\title{
Anti-Coagulation Factor Xa Unit
}

National Cancer Institute

\section{Source}

National Cancer Institute. Anti-Coagulation Factor Xa Unit. NCI Thesaurus. Code C70496.

A unit of anti-factor Xa anticoagulation activity of unfractionated or low molecular weight heparin. 